- A complexity index for root canal treatment is described.

- It was easy to use but incomplete.

- It could be used as a valuable tool in risk management or to select suitable cases of root canal treatment for undergraduates.

- It could also be used to identify the most appropriate setting in which a patient should receive treatment and to allow correlation with treatment outcomes.

\title{
Evaluation of a system for grading the complexity of root canal treatment
}

\author{
A. Muthukrishnan, ${ }^{1}$ J. Owens, ${ }^{2}$ S. Bryant ${ }^{3}$ and P. M. H. Dummer ${ }^{4}$
}

Aim To evaluate the reproducibility of the Restorative Index of Treatment Need (RIOTN) system for grading the complexity of root canal treatment. Methodology The RIOTN system of grading the complexity of root canal treatment was applied to all endodontic referrals to a department of restorative dentistry in a district general hospital within a period of one year. Grading was repeated in 60 randomly selected teeth and weighted kappa analysis was used to test for intra-observer as well as inter-observer agreement with a consultant in restorative dentistry and a vocational trainee (VT).

Results In all, 152 patients were referred for root canal treatment of 186 teeth within the period of study. Weighted kappa for intra-observer agreement when categorising complexity was 0.636 . Weighted kappa for inter-observer agreement with the consultant was 0.570 and that for agreement with the VT was 0.223 .

Conclusion The RIOTN system of grading the complexity of root canal treatment was incomplete; with moderate to poor reproducibility.

\section{INTRODUCTION}

A method of grading complexity in restorative dentistry has been described in the Index of Restorative Dental Treatment Need or RIOTN. ${ }^{1}$ The RIOTN complexity index for root canal treatment in permanent teeth is outlined below with radiographs of selected cases as examples:

Complexity grade 1 - low (Fig. 1 - mandibular right central incisor)

- Single/multiple root canals with curvature $<15^{\circ}$ to the root axis that are considered negotiable from radiographic or

\footnotetext{
${ }^{1 *}$ Associate Specialist in Restorative Dentistry, ${ }^{2}$ Consultant in Restorative Dentistry, Morriston Hospital, Swansea, SA6 6NL; ${ }^{3}$ Research Fellow, ${ }^{4}$ Professor of Restorative Dentistry, Department of Adult Dental Health, School of Dentistry, Wales College of Medicine, Cardiff University, Heath Park, Cardiff, CF14 4XY

${ }^{*}$ Correspondence to: Mrs Akhila Muthukrishnan

Email: akhila.muthukrishnan@swansea-tr.nhs.wales.uk
}

\section{Online article number E26}

Refereed Paper - accepted 25 May 2006

DOI: $10.1038 /$ bdj.2007.170

${ }^{\circledR}$ British Dental Journal 2007; 202: E26 clinical evidence through their entire length. No root canal obstruction or damaged access

- Incision and drainage.

Complexity grade 2 - moderate (Fig. 1 - mandibular left central incisor)

- Single/multiple root canals with curvature $>15^{\circ}$ but $<40^{\circ}$ to the root axis that are considered negotiable from radiographic or clinical evidence through their entire length

- Teeth with incomplete root development.

Complexity grade 3 - high (Fig. 2)

- Single/multiple root canals with curvature $>40^{\circ}$

- Single/multiple root canals that are NOT considered negotiable from radiographic or clinical evidence through their entire length

- Surgical treatment

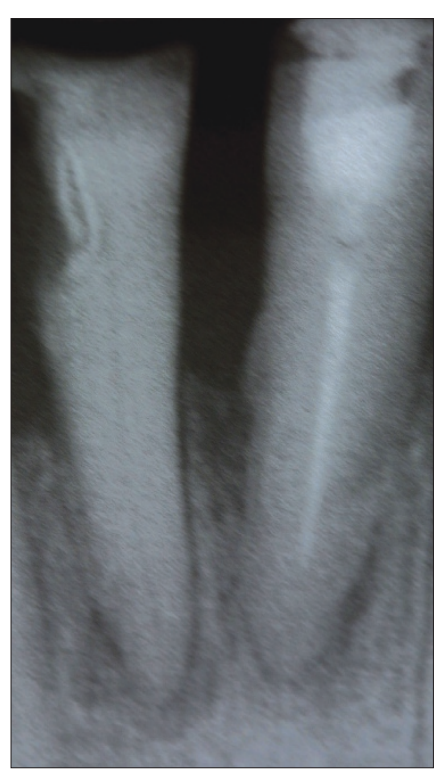

Fig. 1 Mandibular incisors - complexity grades 1 and 2

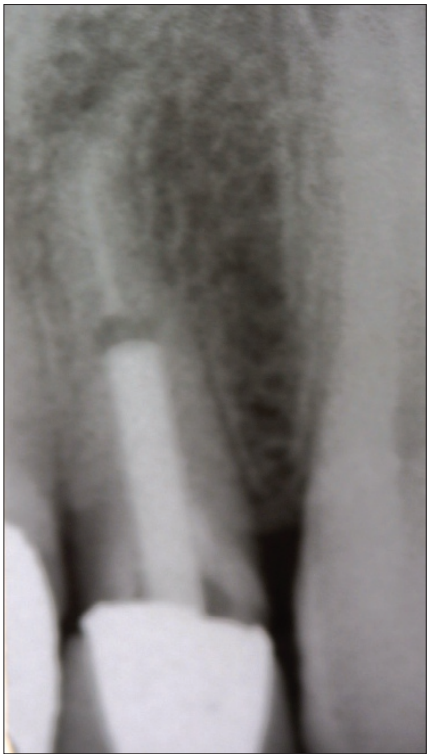

Fig. 2 Maxillary lateral incisor - complexity grade 3 
- Teeth with iatrogenic damage or pathological resorption

- Teeth with difficult root morphology.

A variety of modifying factors are included that have an impact on the complexity grade. The factors are outlined in Table 1.

Despite the fact that the index for grading complexity has been available for some time, it has not been field tested nor any data published on examiner variability when used by different clinicians.

The American Association of Endodontists (AAE) has published a complexity inde ${ }^{2}$ based broadly on patient, diagnostic and treatment considerations. Risk is related on a numeric scale to the degree of complexity as average, high or extreme risk. Though this system is widely advocated and forms part of the guidelines issued by the AAE, no reports on its use have been published.

The Canadian Academy of Endodontics (CAE) has also produced a complexity inde ${ }^{3}$ which provides a numerical value to quantify complexity based on the level of risk assigned. The index is based on patient, tooth and 'additional' considerations. An attempt was made to test its validity but the statistical methods used covered too many variables and the results were not conclusive. ${ }^{4}$

The Dutch Endodontic Treatment Index (DETI) ${ }^{5}$ was devised as a short screening list to assess the risks and difficulty of root canal treatment. If this assessment suggested difficulty, then the Endodontic Treatment Classification (ETC) ${ }^{5}$ could be applied and a numerical value assigned, depending on the level of risk. Both DETI and ETC were found to be useful to assess the difficulty of an endodontic problem and to decide whether to treat or refer to a specialist.

There is a clear need for a practical and user-friendly system of grading complexity of root canal treatment within the UK. Presently, there is no system to guide dentists working in the primary care environment on the appropriateness of referrals for root canal treatment to specialists and consultants. Interestingly, root canal treatment accounted for

Table 1 Modifying factors in the assessment of complexity
Modifying factors that are relevant to root canal treatment
A modifying factor can only increase complexity by one increment. Multiple
factors are not cumulative.
- Coordinated medical (eg renal; cardiac) and/or dental (eg oral surgery;
orthodontic) multi-disciplinary care
- Medical history that significantly affects clinical management (see below)
- Special needs for the acceptance or provision of dental treatment
- Mandibular dysfunction
- Atypical facial pain
- Undiagnosed facial pain
- Presence of a retching tendency
- Limited conventional or surgical operating access
- Endodontic retreatment.
Medical history that significantly affects clinical management
- Patients requiring IM or IV medication as a component of clinical
- management
- Patients with a history of head/neck radiotherapy
- Patients who are significantly immuno-compromised or
- Patients with a significant bleeding dyscrasia/disorder

approximately $18 \%$ of the total number of dento-legal claims in a five-year sample of completed cases between 1996 and $2001 .^{6}$ Thus, there are substantial medico-legal implications for dentists undertaking complex treatment that is beyond their competence.

Root canal treatment is fundamentally about the control of root canal infection through the elimination of micro-organisms and the prevention of re-infection. Much research has focused on the influence of the quality of root canal filling on the outcome of treatment. It has been reported that only $10 \%$ of root fillings undertaken in England and Wales were of a satisfactory technical quality. ${ }^{7}$ A review of cases in Scotland revealed that 54\% of root fillings were not satisfactory. ${ }^{8}$ It is likely that as the complexity of root canal treatment increases, the more difficult it is to achieve high technical standards. However, this issue has not been addressed by previous research.

The aim of this study was to test the reproducibility of the endodontic complexity component of the RIOTN index in terms of intra-observer and inter-observer agreement.

\section{MATERIAL AND METHODS}

The study was based in the Department of Restorative Dentistry, Morriston Hospital, Swansea. Morriston Hospital is a district general hospital in South West Wales, UK.

Data was collected prospectively over a period of one year from May 2001 to June 2002 on data collection forms by five dentists who examined and treated all the patients during this period. A pilot study was conducted initially to validate the methods and refine the format of data collection.

The following details were recorded for each patient:

1. Demographic data, relevant medical history and date of first visit

2. History of present complaint and examination of oral and dental tissues.

The following information was recorded regarding the tooth that was described in the referral:

Tooth number - this was recorded in the FDI format, ie 11, 21 etc. The following signs were noted:

- Tenderness to percussion

- Presence of a sinus

- Tooth mobility

- Deepest pocket probing depth.

Each record was assigned a consecutive database number in order to maintain anonymity for the period of data entry and analysis.

Radiographic examination - Intra-oral periapical radiographs (IOPA) of each tooth were obtained and an OPT where required. IOPAs were obtained using a long cone paralleling technique with a beam aiming device and Rinn film holders (Dentsply UK Limited, Weybridge, UK). A standard buccolingual projection was obtained in all cases and an additional parallax view obtained when further information was required. The X-ray machines used by the department of radiography were the same for all patients (Heliodent $\mathrm{C}^{\circledR}, 70 \mathrm{kV} / 60 \mathrm{kV}, 7 \mathrm{~mA}$, Siemens, Munich, Germany). Kodak Ektaspeed plus ${ }^{\circledR}$ E speed double films (EastmanKodak, Rochester, NY, USA) were used and developed in an 
Table 2 Primary investigator - initial reading and second reading

\begin{tabular}{|l|l|l|l|l}
\hline Complexity grade & Total at initial reading & Percent at initial reading & Total at second reading & Percent at second reading \\
\hline 1 & 3 & $5 \%$ & 4 & $7 \%$ \\
\hline 2 & 24 & $40 \%$ & 30 & $50 \%$ \\
\hline 3 & 33 & $55 \%$ & 26 & $43 \%$
\end{tabular}

automated machine (IGP XR 24, Nova, Dürr ${ }^{\circledR}$ Dental, Beitigham, Germany). Radiographs were viewed in a standard manner using an illuminated viewing box at $3 \mathrm{x}$ magnification and all extraneous light excluded.

The duplicate radiographs obtained enabled the relevant copies to be stored separately from the hospital records. This eased the retrieval process and enabled the assessment of complexity to be made remote from the clinic at a later stage.

\section{Award of complexity grade}

The complexity of root canal treatment was graded by the chief investigator retrospectively on separate forms, based on clinical and radiographic findings. The radiographs were viewed in a standard manner under even illumination with all extraneous light excluded. The reason for assigning a particular complexity grade was recorded and coded. This was undertaken outside clinical time before treatment was initiated and was not modified following treatment. The root canal treatment section of the complexity component of the RIOTN ${ }^{1}$ was used in the assessment of complexity as described.

The following factors were included in the assessment process:

- Radiographic evidence of negotiability of canal/s - this was determined by visually tracing the canal path/s

- Degree of curvature of canal/s - this was measured using a graticule (Peak scale lupe 7x, School of Dentistry, Cardiff University, UK) during the initial assessment by the chief investigator. The subsequent assessments of canal curvature by the chief investigator and those by the consultant and VT were estimations and were not measured using a graticule

- Presence of iatrogenic damage, pathological resorption or abnormal root morphology

- Presence of any modifying factors.

\section{Intra-observer variation}

A total of 60 random numbers were generated out of the total of 186 database numbers. The complexity of root canal treatment was graded again by the chief investigator on a 1-2-3 ordinal scale on separate forms one year after the initial scoring. The examiner was blinded to the complexity grades that had been awarded previously.

\section{Inter-observer variation}

A consultant in restorative dentistry and a vocational trainee who had been qualified for six months undertook the complexity assessment exercise on the same set of 60 cases under similar conditions. Both the observers were trained in the use of the index at the start of the exercise using records and radiographs other than those randomly generated. They were blinded to each other's assessment and also to the original grading carried out by the chief examiner.
Statistical analysis

SPSS (SPSS, Chicago, Illinois, USA) was used to derive simple frequency distributions and cross tabulations. Weighted kappa analyses were carried out to determine the degree of intraobserver and inter-observer agreement.

\section{RESULTS}

The sample consisted of 152 consecutive patients referred to the department between May 2001 and June 2002. This resulted in a sample of 186 teeth. In all, five percent (10) of the total (186) were graded as complexity 1, 48\% (89) of teeth were graded as complexity 2 and $47 \%$ (87) of teeth were graded as complexity 3 for root canal treatment in the initial grading by the principal investigator. Among teeth awarded complexity grade 2, 67 (75\%) were included in this category as they were considered to require revision of previous root canal treatment. Other reasons included incomplete root development (4), canal curvature of $15-40^{\circ}$ (2), and other modifying factors (16).

Among teeth awarded complexity grade 3, 35 (40\%) had iatrogenic damage or pathological resorption. Other reasons included non-negotiable canal (18), difficult root morphology (10), peri-radicular surgery (16), canal curvature greater than $40^{\circ}(2)$ and other modifying factors (6).

\section{Intra-observer variation in the assessment of complexity}

The initial and second readings of the principal investigator were cross-tabulated and are presented in Table 2.

Inter-observer variation in the assessment of complexity

The initial scores of the principal investigator were cross tabulated against the scores of the consultant and vocational trainer and are presented in Tables 3 and 4 respectively. The kappa analysis of all the results is presented in Table 5.

\section{DISCUSSION}

The aim of the study was to evaluate the reproducibility of the Restorative Index of Treatment Need (RIOTN) system for grading the complexity of root canal treatment. It was felt important to select clinicians with different levels of experience in order to test the index for inter-observer variation, since the perception of complexity may differ as a result of clinical knowledge. The data was derived from all endodontic referrals to a department of restorative dentistry in a district general hospital within a period of one year and thus was not biased in terms of any selection criteria.

The conditions for radiographic examination were standardised for the purpose of this study. However, it is well recognised that the radiographic interpretation of dental radiographs is subject to intra-observer and inter-observer variations. ${ }^{5,9,10}$ For example, the mandibular left central incisor in Figure 1 has been graded as complexity 2 due to the 
Table 3 Author vs consultant cross tabulation

\begin{tabular}{l|l|l|l|l|l}
\hline \multirow{2}{*}{$\begin{array}{l}\text { Principal } \\
\text { investigator }\end{array}$} & \multicolumn{4}{|l|}{ Consultant reading } & \multirow{2}{*}{ Total } \\
\cline { 2 - 7 } & 1 & 2 & 3 & 9 (not assessable) & \\
\hline 1 & 2 & - & 1 & - & 3 \\
\hline 2 & 2 & 12 & 7 & 3 & 24 \\
\hline 3 & - & 5 & 27 & - & 32 \\
\hline Total & 4 & 17 & 35 & 3 & 59 \\
\hline Not recorded: 1 & & & & & \\
\hline
\end{tabular}

\begin{tabular}{l|l|l|l|l|} 
Table 4 Author vs VT cross tabulation \\
\hline \multirow{2}{*}{ Principal investigator } & \multicolumn{3}{|l|}{ VT reading } & \multirow{2}{*}{ Total } \\
\cline { 2 - 5 } & 1 & 2 & 3 & \\
\hline 1 & 2 & & 1 & 3 \\
\hline 2 & 10 & 1 & 11 & 22 \\
\hline 3 & 6 & 6 & 20 & 32 \\
\hline Total & 18 & 7 & 32 & 57 \\
\hline Not recorded: 3 & & & &
\end{tabular}

Table 5 Weighted kappa analysis

\begin{tabular}{|c|c|c|c|}
\hline & Author vs author re-read & Author vs consultant & Author vs VT \\
\hline \multicolumn{4}{|c|}{ Weighted kappa with linear (Cichetti-Allison) weights } \\
\hline Observed weighted agreement & 0.867 & 0.857 & 0.640 \\
\hline Expected & 0.692 & 0.700 & 0.586 \\
\hline Weighted kappa & 0.568 & 0.523 & 0.131 \\
\hline \multicolumn{4}{|c|}{ Weighted kappa with quadratic (Fleiss-Cohen) weights } \\
\hline Observed weighted agreement & 0.933 & 0.920 & 0.759 \\
\hline Expected & 0.817 & 0.813 & 0.690 \\
\hline Weighted kappa & 0.636 & 0.570 & 0.223 \\
\hline
\end{tabular}

modifying factor of retreatment. However, the radiographic interpretation of the negotiability of the apical third of the canal may vary.

It is possible that the assessment of the angle of canal curvature could be incorrect, as they were estimations during the reassessment process and not accurate measurements. However, if such an index is to be used by a generalist to aid in the decision whether to treat or refer, then realistically, the angle would be estimated rather than accurately measured in general practice.

The complexity indices in use by the AAE, CAE and the Dutch ETC all have one thing in common: the ability to assign a cumulative numerical value, which increases with the degree of complexity. However, the RIOTN lacks the ability to provide a cumulative score. For example, in Figure 2, the maxillary lateral incisor appears to have an angle of canal curvature $>40^{\circ}$. It also contains a post and has a modifying factor of retreatment. However, the RIOTN can only award it the maximum complexity of 3.

In general, the system of grading complexity used in this study was found to be rapid and easy to use. The draw-backs of this classification system are summarised below:

- Assessment of canal negotiability is ambiguous and subjective. For example, one of the criteria for awarding a complexity grade 1 is as follows: 'Single/multiple root canals with curvature $<15^{\circ}$ to the root axis that are considered negotiable from radiographic or clinical evidence through their entire length. No root canal obstruction or damaged access.' This would appear to suggest radiographic assessment prior to treatment as well as clinical assessment during treatment; this implies the score could change. The CAE, ETC and AAE are less ambiguous in this regard. They base their assessment of the canal on radiographic visibility only

- There is potential for variable interpretation of 'difficult' root morphology. The AAE, CAE and ETC systems of grading complexity all define various aspects of root morphology further and score them appropriately based on the risk assessment. For example, in the CAE system, the highest score of five units is assigned to any of the following findings: canal path into C or S form; canal curvature $>30^{\circ}$; molar with four canals. It could be argued that the RIOTN system is too simplistic and is unable to take account of canal complexities, other than angle of curvature

- There is potential for variable estimation of the angle of canal curvature. The AAE, ETC and CAE use different categorisation with regards to canal curvature, ie $<10^{\circ}, 10^{\circ}-30^{\circ}$ or $>30^{\circ}$. It is possible that these angles are easier to assess subjectively than $15^{\circ}$ and $40^{\circ}$. However, this issue has not been addressed by previous research. In this study, only two out of 89 cases in complexity grade 2 and two out of 87 cases in complexity grade 3 were categorised as such due to their angle of curvature. Of course, this is only one aspect of canal anatomy that may influence treatment; others include the radius of curvature and the classification of the canal system

- There can be variable interpretation of 'difficult access'. In contrast, the ETC system assigns the highest complexity score of five units when the mouth opening is less than $25 \mathrm{~mm}$

- The RIOTN provides an unclear interpretation of "co-ordinated medical history'. The AAE, CAE and ETC all use the ASA $^{11}$ (American Association of Anesthesiology) classification which quantifies the degree of risk with regards to the medical history

- It is not quantifiable

- It is confusing because it combines root canal treatment and surgical treatment

- It has not to date been tested and accepted by an international body of opinion. 
In conclusion, within the limitations of this study, the RIOTN system of grading the complexity of root canal treatment was found to be incomplete, with moderate to poor reproducibility.

AM would like to sincerely thank Mr Karl Bishop, Consultant in Restorative Dentistry, Morriston Hospital, for his guidance in setting up the data collection forms which were used as part of my MPhil thesis. She would also like to thank the staff in the department for their help with data collection.

1. Falcon F C, Richardson P, Shaw M J, Bulman J S, Smith B G N. Developing an index of restorative dental treatment need. Br Dent $J$ 2001; 190: 479-486.

2. Curtis E K. Simon D C. Endodontic case difficulty assessment: the team approach Gen Dent 1999; 47: 340-344.

3. Canadian Academy of Endodontics. Standards of Practice. Edmonton, Canada: Canadian Academy of Endodontics, 1998.

4. Morand M A. Reliability study of a new evaluation tool in endodontics. J Dent
Educ 1992; 56: 63 (abstract \#117).

5. Ree M H, Timmerman M F. Wesselink P R. An evaluation of the usefulness of two endodontic case assessment forms by general dentists. Int Endod J 2003: 36: 545-555.

6. Dental Protection Limited. Riskwise. London: Dental Protection Ltd, 2003.

7. Dummer P M H. The quality of root canal treatment provided by general dental practitioners working within the general dental services in England and Wales. Dent Profile 1998; 19: 8-10.

8. Saunders W P, Saunders E M, Sadiq J, Cruickshank E. Technical standard of root canal treatment in an adult Scottish population. Br Dent J 1997; 182: $382-386$

9. Seltzer S, Bender L B. Cognitive dissonance in endodontics. Oral Surg Oral Med Oral Pathol 1965; 20: 506-516.

10. Goldman M. Reliability of interpretation of dental radiographs. J Dent Res 1982; 61: 239 .

11. Sacklad M. Grading of patients for surgical procedures. Anaesthesiol 1941; 2: 281-284. 